\title{
Do surgeons really know how to perform neuromonitoring in thyroid surgery? An awareness study
}

\author{
Ioannis Pliakos, Theodosios S. Papavramidis \\ $1^{\text {st }}$ Propedeutic Department of Surgery, AHEPA University Hospital, Aristotle University of Thessaloniki, Thessaloniki, Greece \\ Contributions: (I) Conception and design: TS Papavramidis; (II) Administrative support: I Pliakos; (III) Provision of study materials or patients: TS \\ Papavramidis; (IV) Collection and assembly of data: TS Papavramidis; (V) Data analysis and interpretation: I Pliakos; (VI) Manuscript writing: All \\ authors; (VII) Final approval of manuscript: All authors. \\ Correspondence to: Ioannis Pliakos, MD, PhD. $1^{\text {st }}$ Propedeutic Department of Surgery, AHEPA University Hospital, Aristotle University of \\ Thessaloniki, Aigaiou 6, 54655, Thessaloniki, Greece. Email: plliakos@hotmail.com.
}

\begin{abstract}
Background The aim of this questionnaire-based observational study is to examine the awareness of endocrine surgeons regarding the use of intraoperative neuromonitoring (IONM) techniques in thyroid surgeries.

Methods: A survey project based on a structured questionnaire was conducted during the $14^{\text {th }}$ Spring Meeting of the Greek Society of Endocrine Surgeons (GSES). The questionnaire consisted of 14 items and was handed to all participants—by the key speaker—during the neuromonitoring session.

Results: In the $14^{\text {th }}$ spring meeting of GSES there were 205 delegates present. Among them there were 35 residents and 6 medical students. The surgeons being potential responders were 119. In the session of IONM, there were 83 eligible surgeons present and the questionnaire was answered voluntarily by 59 of them (71.08\%). The responders' group consisted of 26 members of the GSES and 33 non-members.

Conclusions: Most of the participants believed that there are many benefits in the use of IONM such as anatomically identifying the branches of the laryngeal nerve, monitoring their functionality or offering to patients' greater confidence to the surgery. Attention should be paid not to abandon the conventional techniques for identifying the laryngeal nerve, as IONM may not always be available. Evidence is needed to establish the proper indications for its application.
\end{abstract}

Keywords: Thyroid surgery; intraoperative neuromonitoring (IONM); surgeons' survey

Submitted Jun 24, 2020. Accepted for publication Dec 13, 2020.

doi: $10.21037 /$ gs-20-579

View this article at: http://dx.doi.org/10.21037/gs-20-579

\section{Introduction}

Surgeons operating on thyroid glands aim mainly at conducting a safe and oncologically sound surgery. A major concern is the iatrogenic injury of the recurrent laryngeal nerve (RLN), as it is a serious complication resulting in severe morbidity. The incidence of temporary and permanent vocal cord palsy after thyroid surgery has been reported to range from $2.0 \%$ to $7.2 \%$ and $0.2 \%$ to $0.9 \%$, respectively (1-3). Symptomatic RLN paralysis constitutes a major cause of impaired quality of life and a factor of job incompetence, postoperatively (4). To minimize RLN related complications, neuromonitoring (continuous or intermittent) is employed, especially by low volume surgeons (with proven benefit) or in extreme conditions and reoperations (3). In 2011, the International Neural Monitoring Study Group proceeded to an international standards guideline statement regarding the standardization of the intraoperative neuromonitoring (IONM) procedure (5). Their statement includes recommendations regarding the equipment setup, the anesthesia, the placement of endotracheal tube, the evaluation of intraoperative loss of signal and finally the waveform definition and assessment. The above represents a valuable 


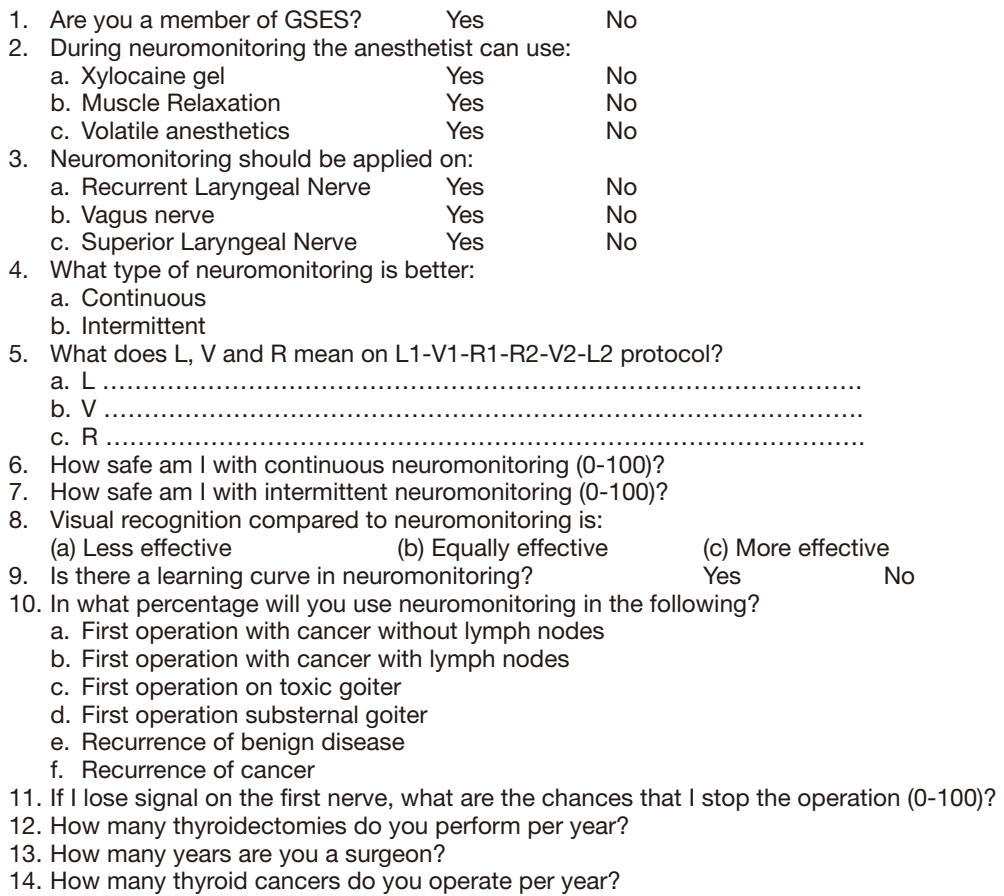

Figure 1 Perception of visual recognition $v s$. IONM effectiveness between members and non-members of GSES. IONM, intraoperative neuromonitoring; GSES, Greek Society of Endocrine Surgeons.

"manual" for those being fond of employing IONM during thyroid surgery, and helps to standardize the procedures, establish a global "language" regarding IONM and minimize the possibility of bias $(5,6)$.

The aim of this questionnaire-based observational study is to examine the awareness of endocrine surgeons regarding the use of IONM techniques in thyroid surgeries.

We present the following article in accordance with the SURGE reporting checklist (available at http://dx.doi. org/10.21037/gs-20-579).

\section{Methods}

This survey project was conducted during the $14^{\text {th }}$ Spring Meeting of the Greek Society of Endocrine Surgeons (GSES), held in Athens, Greece. This was an extended meeting, involving many surgeons that perform endocrine surgery and aiming at issuing recommendations for clinical practice. Such a recommendation, concerning IONM, was published in Hellenic Fournal of Surgery. The questionnaire was designed to collect data on IONM usage patterns among Greek surgeons performing thyroidectomies. Participation was voluntary and anonymous. The survey was completed with a structured questionnaire including both multiple-choice and free text questions. The questionnaire consisted of 14 items and was handed to all participantsby the key speaker-during the neuromonitoring session. Surveys were collected at the end of the session. A translated sample of the questionnaire is shown in Figure 1.

\section{Statistical analysis}

The data were analyzed using standard statistical methods. Descriptive statistics, including means, ranges, and standard deviations were used. The Mann-Whitney U test and Student $t$-test were used to determine statistical significance $(\mathrm{P}<0.05)$. A statistical analysis was performed with the SPSS 25.0 data analysis program (SPSS Inc., Chicago, IL, USA).

\section{Ethical statement}

The study was conducted in accordance with the Declaration of Helsinki (as revised in 2013). The study did not involve human experiments, therefore no approval from the university's ethical board was required. For the same reason, we did not obtain an inform consent from the participants. 


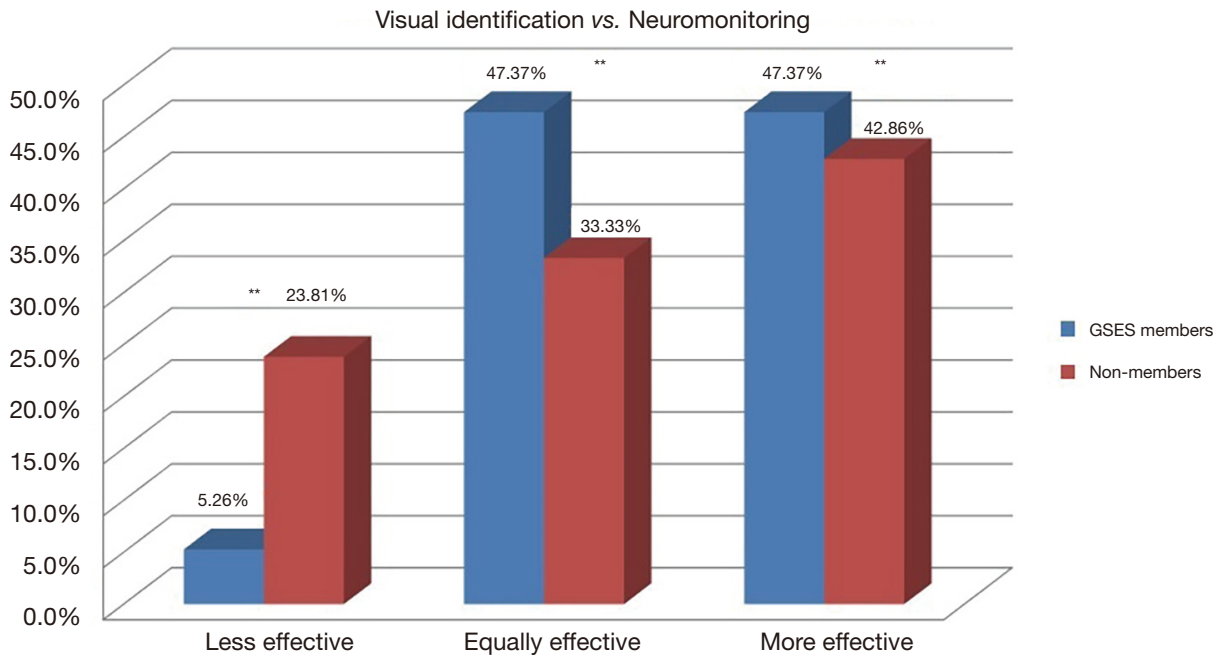

Figure 2 Comparison of perceptions of GSES members and non-members about visual recognition vs. neuromonitoring. GSES, Greek Society of Endocrine Surgeons.

\section{Results}

In the $14^{\text {th }}$ spring meeting of GSES there were 205 delegates present. Among them there were 35 residents and 6 medical students. Of the 164 experts, there were 18 endocrinologists, 9 registered nurses, 4 internal medicine physicians, 3 anesthesiologists, 3 pediatric surgeons, 1 general practitioner, 1 gynecologist, 1 radiologist and 5 physicians that did not specify their specialty. The surgeons being potential responders were 119. In the session of IONM, there were 83 eligible surgeons present and the questionnaire was answered voluntarily by 59 of them (71.08\%). The responders' group consisted of 26 members of the GSES and 33 non-members.

First question was about membership or not of GSES. The second question asked which medication could be used in the anesthetic protocol, without causing any problems to the IONM. Xylocaine gel was judged inappropriate for IONM by $70.0 \%$ of the participants. The respective percentages were $87.9 \%$ for muscle relaxants and $2.9 \%$ for volatile anesthetics. There was no statistically significant difference between GSES members and non-members.

The third question asked which nerve IONM should be applied to. Eighty-four point two percent of the participants answered the RLN, $69.4 \%$ the vagus nerve and $27.6 \%$ the superior laryngeal nerve (SLN). There was no statistically significant difference between members and non-members concerning RLN and vagus, although non-members believed that SLN should be monitored while members did not $[39.4 \%$ vs. $7.7 \%(\mathrm{P}<0.01)]$.

The fourth question attempted to identify the type of IONM that was the best, according to surgeons' opinion. Sixty-eight point three percent believed that continuous IONM was the best. There was no statistically significant difference between GSES members and non-members.

The fifth question tried to identify how many participants knew what the initials $\mathrm{L}, \mathrm{V}$ and $\mathrm{R}$ stood for in the L1-V1-R1-R2-V2-L2 protocol. Interestingly enough, only $27.3 \%$ of the participants gave a correct answer, with no difference between members and non-members.

The following two questions tried to find if the surgeons felt safe, either with continuous or with intermittent IONM. In the first case, $74.5 \%$ of the participants answered that they felt safe with continuous IONM, while the same percentage for intermittent neuromonitoring was $65.2 \%$. The members of GSES felt safer when IONM was available at $69.2 \%$ and $52.6 \%$ (for continuous and intermittent IONM respectively), while the non-members felt safer at $77.3 \%$ and $71.0 \%$, respectively.

The eighth question asked the participants to compare the effectiveness of visual recognition compared to IONM. Fifteen percent believed that visual recognition was less effective, $40.0 \%$ believed that it was equally effective, while $45.0 \%$ believed that it was more effective. However, there was a vast difference in the perception of visual recognition vs. IONM between members and non-members, as displayed in Figure 2 ( $\mathrm{P}<0.01$ for all values).

Most of the participants (97.6\%) believed that there was 
Table 1 Probability of IONM use according to GSES questionnaire

\begin{tabular}{|c|c|c|c|c|}
\hline Condition & Total \% $(\mathrm{N}=59)$ & $\begin{array}{l}\text { GSES-members \% } \\
(n=26)\end{array}$ & $\begin{array}{c}\text { Non-members \% } \\
(n=33)\end{array}$ & $P$ \\
\hline First operation on thyroid cancer without lymph nodes & 57.6 & 40.8 & 68.3 & 0.01 \\
\hline First operation on thyroid cancer with lymph nodes & 77.9 & 69.6 & 83.2 & 0.01 \\
\hline Toxic goiter & 58.0 & 44.2 & 67.2 & 0.01 \\
\hline Recurrence of cancer & 90.9 & 83.1 & 96.3 & 0.01 \\
\hline Total & 72.3 & 61.3 & 79.6 & 0.01 \\
\hline
\end{tabular}

IONM, intraoperative neuromonitoring; GSES, Greek Society of Endocrine Surgeons.

a learning curve in IONM.

In the tenth question, the questionnaire tried to determine the probability of using IONM in various conditions. Table 1 displays the data for each condition and analyzes it for GSES members and non-members.

Eleventh question showed that $56.5 \%$ of the participants would stop the operation upon signal loss intraoperatively. The above mentioned was independent of being a member of GSES or not, since $46.5 \%$ of non-members $v s .39 .5 \%$ of members would stop the operation $(\mathrm{P}=\mathrm{ns})$.

The twelfth question showed that the mean volume of thyroidectomies is 73 per surgeon per year. However, figures changed when distinguishing between GSES members and non-members. The former performs an average of 143 thyroidectomies per year, while the latter perform 23 thyroidectomies per year $(\mathrm{P}<0.01)$.

The thirteenth question showed that the participants' mean duration of surgery practice was 13 years (members vs. non-members 15 vs. 12, $\mathrm{P}=\mathrm{ns}$ ).

Finally, the fourteenth question showed that the average number of thyroid malignancies operated per surgeon per year was 26.5. However, GSES members operated an average of 53 cases per year, while non-members operated 8 cases per year $(\mathrm{P}<0.01)$.

\section{Discussion}

The avoidance of the RLN injury during thyroidectomy operations is of high importance, not only due to the reported high risk of the procedure, but also due to the severe lifelong disability it imposes. It is referred in literature that a temporary damage of the RLN can be seen in up to $7.2 \%$ of the performed surgeries, while the same percentage is $0.9 \%$ for permanent lesions $(1,2)$. Moreover, the damaging of the RLN, with the consequent voice loss, could have serious implications to the individual's life routine, job and psychology. An observational study, surveying among others the attempt of endocrine surgeons to preserve branches of the laryngeal nerve and their perception of its importance, reports that $90 \%$ of them try to preserve it, although only half of them try to identify it (7). Endocrine surgeons reported that they find it very important to preserve the RLN and they usually employ IONM for this purpose. IONM is a relatively modern technique that allows the real-time identification of the branches of the laryngeal nerves and the confirmation of their proper functionality. It is commonly used in the surgical setting worldwide, with figures that reach $95 \%$ of the cases (8). In a study from Italy, it is reported that almost half $(48 \%)$ of the IONM procedures are performed in public hospitals (9). This fact is opposed to the national data in Greece, where the public hospitals are still naive to its use, as far as our experience is concerned.

In our observational study, we evaluated the level at which this technique is used and the characteristics and belief of the surgeons applying it. The participants reported that they would be willing to use IONM in 57.6-90.9\% of their surgeries, depending on their type. The surgery types that would mostly require the application of this method were the recurrence of thyroid cancer (90.9\%), the recurrence of a benign disease $(86.1 \%)$ and the operation for thyroid cancer with positive neck lymph nodes (77.9\%). However, it is rather questionable whether IONM is truly applied at these percentages, particularly in public hospitals, as the economic circumstances and the insufficient education of the surgeons discourage its use. Although, 
most participants would employ IONM for their surgeries, $85 \%$ of them find this method equally or less effective than visual recognition for identifying the RLN. This is probably a result of their training in the latter method and their unfamiliarity with the former. This hypothesis is also reinforced by the fact that almost all of the participants believe that there is a learning curve in the use of IONM, meaning that significant time and effort are required for mastering and using this technique safely. The majority of the participants believe that the continuous version of IONM is more effective than the intermittent one, with the continuous offering a feeling of greater safety. However, they lack training in these techniques, as indicated by the question referring to their knowledge of " $\mathrm{L}$ ", " $\mathrm{V}$ " and " $\mathrm{R}$ " symbols. To our surprise, only $27.3 \%$ of the participants answered correctly. When IONM cannot be used correctly, research on the ineffectiveness or effectiveness of IONM for thyroid surgery should be cautiously interpreted. Regardless of whether the result is positive or negative, incorrect use of IONM increases burden in thyroid surgery.

In the literature, the motives for applying this strategy are reported. It is referred that in most cases IONM is used for legal reasons, while both RLN confirmation and RLN identification constitute the actual reasons in only $20 \%$ of the cases (9). In another prospective study, the feeling of the patients concerning the use of this method was evaluated. It was found that the anxiety of the patients the day before surgery was decreased, while their confidence about the procedure and their trust in their doctor were both raised, when using IONM (10). This seems to add value to the use of this technique, not only for the safer and more effective conduction of the surgery, but also for patients' psychological reasons. Younger surgeons are more likely to use this method, both in literature and in our study (8). In our study, a relatively high percentage of surgeons (56.5\%) would continue the procedure if the signal of the nerve was lost intraoperatively, something that comes in agreement with other studies (11). Experienced surgeons (namely the ones that would continue the procedure) trust visual recognition more than neuromonitoring, as they know, even in the lack of valid relevant studies, that the latter method comes with a high percentage of false negative results (loss of signal of an intact and functioning nerve).

Among the members of GSES there are surgeons with the widest experience and with the greatest surgical volume in thyroid surgery in our country. The Greek Society of Endocrine Surgery, as well as other endocrine surgery societies from all over Europe, has issued guidelines, according which an experienced surgeon does not benefit from the use of neuromonitoring and thus is not obliged to use it.

\section{Conclusions}

There seem to be many benefits in the use of IONM. First of all, it seems to be a reliable tool not only for anatomically identifying the branches of the laryngeal nerve, but also for monitoring their functionality. Moreover, it satisfies the patients, offering them greater confidence. This could result in higher trust in their doctors and could contribute to their consent for surgery when it is needed. Unfortunately, there seems to be a learning curve for its use and significant training is required. This would currently be hard to achieve in the Greek educational environment where the economic circumstances pose restrictions. Moreover, attention should be paid not to abandon the conventional techniques for identifying the laryngeal nerve, as IONM may not always be available for technical reasons. The cost for its purchase and maintenance could also be an issue that has to be evaluated. More prospective randomized studies which will evaluate the clinical benefit of correctly performed IONM against more traditional techniques, as well as its overall costeffectiveness, have to be conducted. Evidence is needed to establish the proper indications for its application.

\section{Acknowledgments}

Funding: None.

\section{Footnote}

Reporting Checklist: The authors have completed the SURGE reporting checklist. Available at http://dx.doi. org/10.21037/gs-20-579

Data Sharing Statement: Available at http://dx.doi. org/10.21037/gs-20-579

Peer Review File: Available at http://dx.doi.org/10.21037/gs20-579

Conflicts of Interest: Both authors have completed the ICMJE uniform disclosure form (available at http://dx.doi. org/10.21037/gs-20-579). The authors have no conflicts of interest to declare. 
Ethical Statement: The authors are accountable for all aspects of the work in ensuring that questions related to the accuracy or integrity of any part of the work are appropriately investigated and resolved. The study was conducted in accordance with the Declaration of Helsinki (as revised in 2013). The study did not involve human experiments, therefore no approval from the university's ethical board was required. For the same reason, we did not obtain an inform consent from the participants.

Open Access Statement: This is an Open Access article distributed in accordance with the Creative Commons Attribution-NonCommercial-NoDerivs 4.0 International License (CC BY-NC-ND 4.0), which permits the noncommercial replication and distribution of the article with the strict proviso that no changes or edits are made and the original work is properly cited (including links to both the formal publication through the relevant DOI and the license). See: https://creativecommons.org/licenses/by-nc-nd/4.0/.

\section{References}

1. Lo CY, Kwok KF, Yuen PW. A prospective evaluation of recurrent laryngeal nerve paralysis during thyroidectomy. Arch Surg 2000;135:204-7.

2. Steurer M, Passler C, Denk DM, et al. Advantages of recurrent laryngeal nerve identification in thyroidectomy and parathyroidectomy and the importance of preoperative and postoperative laryngoscopic examination in more than 1000 nerves at risk. Laryngoscope 2002;112:124-33.

3. Koulouris C, Papavramidis TS, Pliakos I, et al. Intraoperative stimulation neuromonitoring versus intraoperative continuous electromyographic neuromonitoring in total thyroidectomy: identifying laryngeal complications. Am J Surg 2012;204:49-53.

Cite this article as: Pliakos I, Papavramidis TS. Do surgeons really know how to perform neuromonitoring in thyroid surgery? An awareness study. Gland Surg 2021;10(1):201-206. doi: $10.21037 / g s-20-579$
4. Smith E, Taylor M, Mendoza M, et al. Spasmodic dysphonia and vocal fold paralysis: outcomes of voice problems on work-related functioning. J Voice 1998;12:223-32.

5. Randolph GW, Dralle H, Abdullah H, et al. Electrophysiologic recurrent laryngeal nerve monitoring during thyroid and parathyroid surgery: international standards guideline statement. Laryngoscope 2011;121 Suppl 1:S1-16.

6. Dralle H, Sekulla C, Lorenz K, et al. Loss of the nerve monitoring signal during bilateral thyroid surgery. $\mathrm{Br} \mathrm{J}$ Surg 2012;99:1089-95.

7. Almquist M, Nordenström E. Management of the exterior branch of the superior laryngeal nerve among thyroid surgeons - Results from a nationwide survey. Int J Surg 2015;20:46-51.

8. Henry BM, Graves MJ, Vikse J, et al. The current state of intermittent intraoperative neural monitoring for prevention of recurrent laryngeal nerve injury during thyroidectomy: a PRISMA-compliant systematic review of overlapping meta-analyses. Langenbecks Arch Surg 2017;402:663-73.

9. Dionigi G, Lombardi D, Lombardi CP, et al. Intraoperative neuromonitoring in thyroid surgery: a point prevalence survey on utilization, management, and documentation in Italy. Updates Surg 2014;66:269-76.

10. Babińska D, Barczyński M, Osęka T, et al. Comparison of perioperative stress in patients undergoing thyroid surgery with and without neuromonitoring - a pilot study. Langenbecks Arch Surg 2017;402:719-25.

11. Khamsy L, Constanthin PE, Sadowski SM, et al. Loss of neuromonitoring signal during bilateral thyroidectomy: no systematic change in operative strategy according to a survey of the French Association of Endocrine Surgeons (AFCE). BMC Surgery 2015;15:95. 